\title{
Evaluating Different Scenarios in Wanets to Find Shortest Path Applying ACO
}

\author{
Dr. Gagandeep Jagdev \\ Dept. of Computer Science, Punjabi University Guru Kashi College, Damdama Sahib, Punjab, India. \\ *Corresponding Author Dr. Gagandeep Jagdev, Dept. of Computer Science, Punjabi University Guru \\ Kashi College, Damdama Sahib, Puniab, India.
}

\begin{abstract}
The purpose of the routing protocols in networks is to find the shortest path for transferring the data and enhancing the overall throughput of the network. The paper emphasizes on conducting practical implementation of ACO algorithm to find out the shortest path under different scenarios differentiating in number of iterations, number of ants, and evaporation rate. The implemented algorithm may be used in large network with heavy loads in selecting the most appropriate path.Moreover, with the increase in the number of iterations, the chances of controlling the congestion effectively also increases.
\end{abstract}

Keywords:Ants, ACO, evaporation rate, iterations, shortest path

\section{INTRODUCTION}

Each ant in the ant colony has its own job which it should be intended to perform. The task performed by different ants are integrated in such a manner that overall capability of solving complex problems is enhanced. The survival related problems like opting for shortest walking path, finding and storing food are handled by ant colony without any supervision. Ants observe pheromone trails to exchange and broadcast information regarding which path to follow. The tendency of ants is to follow the shortest path in order to make more trips and deliver food to the colony. More the ants follow a particular trail, more are the chances of following ants to follow the same path. This process is termed as positive feedback loop and the probability of an ant choosing a path is proportional to the number of ants which have already passed through that particular path. The research is been carried out to simulate the natural behavior of ants to solve real world problems like TSP (Travelling salesman problem) and data mining.

ACO is intended to solve the problems based on below mentioned concepts.

- Each and every path adopted by the ant has an association with the candidate solution to a given problem under study.

- Whenever an ant opts for a path, it drops several pheromone on that particular path in proportion with the worth of the corresponding candidate solution for the target problem.

- The path having more number of pheromones is more likely to be selected by the other ants.

\section{CONTRIBUTION AND IMPLEMENTATION}

This section describes the scenarios and reading obtained in accordance with finding shortest route length under different circumstances. The details of the parameters considered in doing so are given below.

Initial parameters of $\mathrm{ACO}$

- $\quad$ Maximum number of iterations (maxIter $=500)$

- Maximum number of ants $($ antNo $=50)$

- Initial pheromone concentration [tau0 $=10 * 1 /($ graph.n $*$ mean( graph.edges(:) ) )]

- Pheromone matrix [tau $=\operatorname{tau} 0 *$ ones $($ graph.n, graph.n)] 
- Desirability of each edge [eta $=1 . /$ graph.edges $]$

- $\quad$ Evaporation rate $[$ rho $=0.5]$

- Pheromone exponential parameters [alpha $=1]$

- Desirability exponential parameter $[$ beta $=1]$

An interface has been designed which have been fragmented in three sub sections; number of iterations, best tour, and displaying all pheromones.

\section{Case1}

maxIter $=500$

antNo $=50$

rho $=0.5$

alpha $=1$

beta $=1$
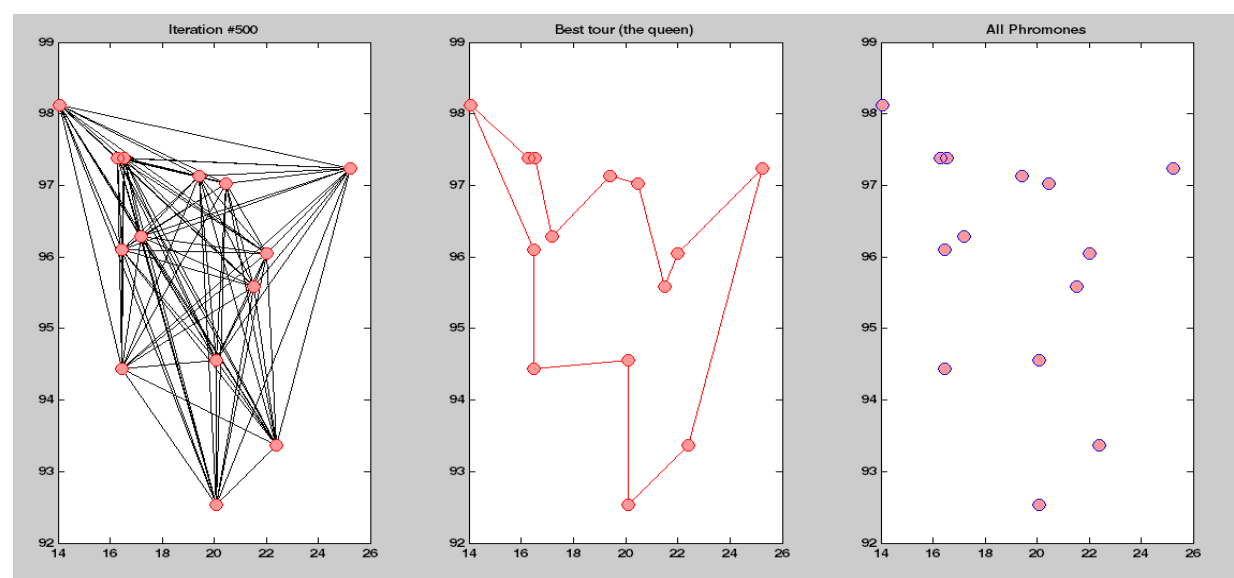

Fig1.The figure shows the formed iterations, best tour, and all pheromones as per reading of Case1

The shortest length in case 1 of 500 iterations with 50 ants at evaporation 0.5 comes out to be 30.8785 as shown by the readings obtained in Fig2.

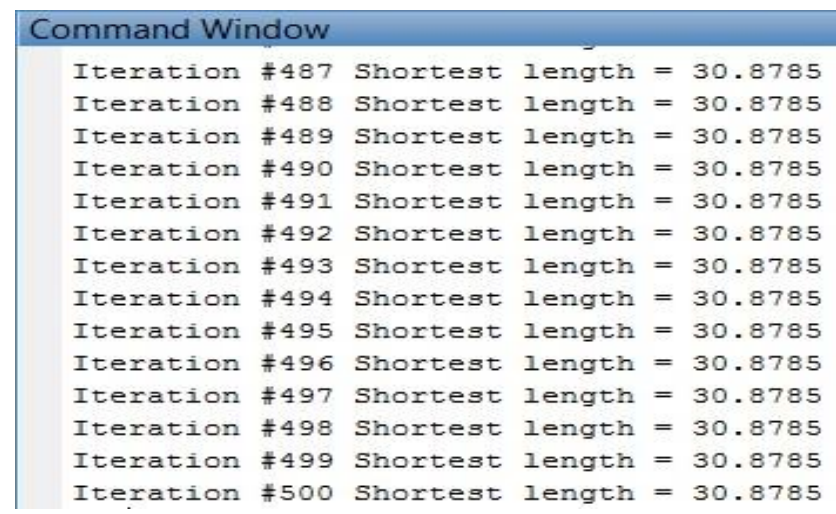

Fig2.The shortest length in case 1 of 500 iterations with 50 ants at evaporation 0.5 comes out to be 30.8785

\section{Case2}

maxIter $=100$

antNo $=50$

rho $=0.5$

alpha $=1$

beta $=1$ 


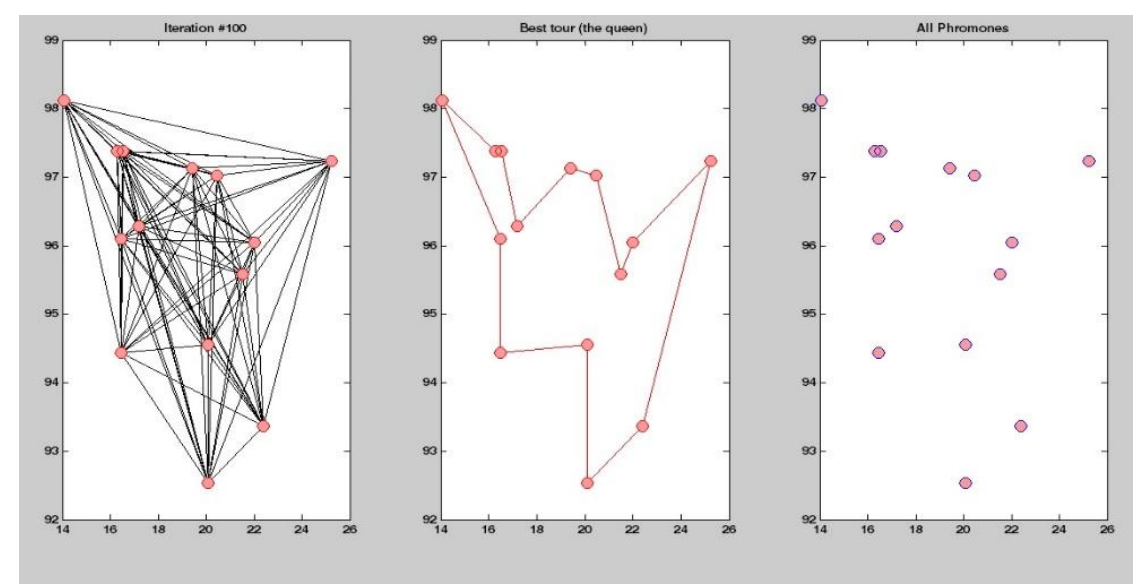

Fig3. The figure shows the formed iterations, best tour, and all pheromones as per reading of case 2

The shortest length in case of 100 iterations with 50 ants at evaporation 0.5 comes out to be 30.8785 as shown in Fig4.

Command Window
Iteration $\$ 487$ shortest length $=30.8785$
Iteration $\$ 488$ shortest length $=30.8785$
Iteration $\$ 489$ shortest length $=30.8785$
Iteration $\$ 490$ shortest length $=30.8785$
Iteration $\$ 491$ shortest length $=30.8785$
Iteration $\$ 492$ shortest length $=30.8785$
Iteration $\$ 493$ shortest length $=30.8785$
Iteration $\$ 494$ shortest length $=30.8785$
Iteration $\$ 495$ shortest length $=30.8785$
Iteration $\$ 496$ shortest length $=30.8785$
Iteration $\$ 497$ Shortest length $=30.8785$
Iteration $\$ 498$ shortest length $=30.8785$
Iteration $\$ 499$ shortest length $=30.8785$
Iteration $\$ 500$ shortest length $=30.8785$

Fig4.The shortest length in case 2 of 100 iterations with 50 ants at evaporation 0.5 comes out to be 30.8785

\section{Case3}

maxIter $=50$

antNo $=50$

rho $=0.5$

alpha $=1$

beta $=1$

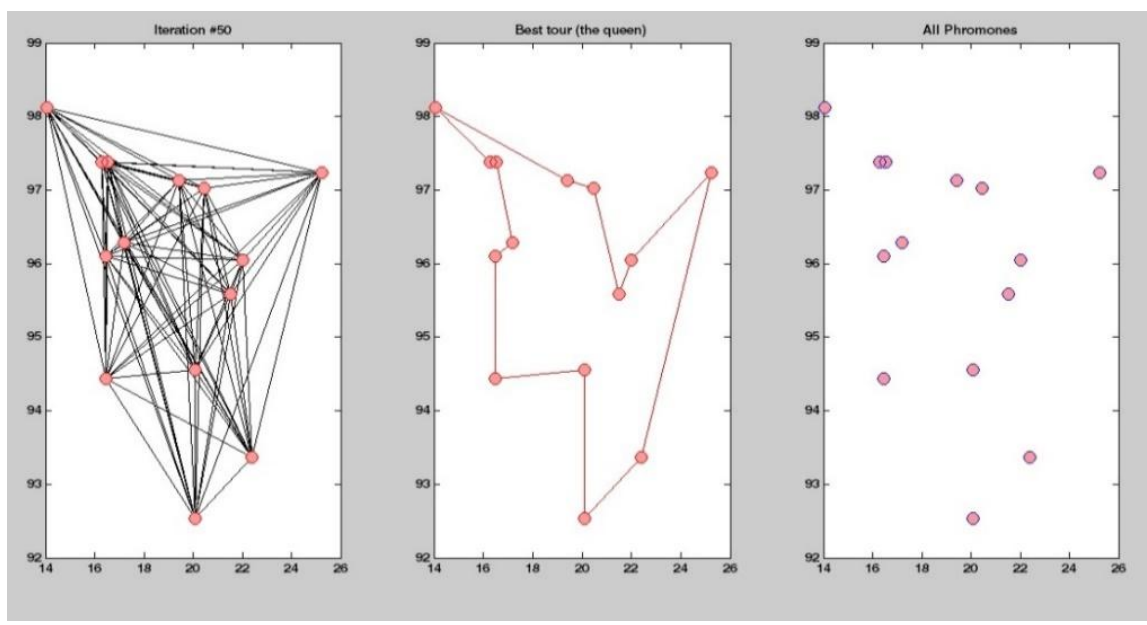

Fig5.The figure shows the formed iterations, best tour, and all pheromones as per reading of case 3

The shortest length in case of 50 iterations with 50 ants at evaporation 0.5 comes out to be 31.567 as shown in Fig 6. 


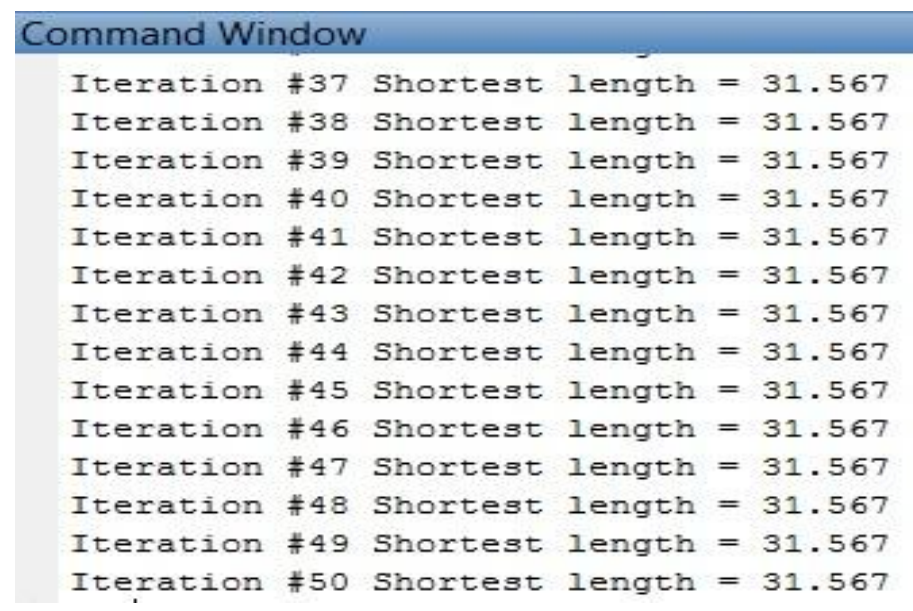

Fig6. The shortest length in case 3 of 50 iterations with 50 ants at evaporation 0.5 comes out to be 31.567

\section{Case 4}

maxIter $=500$

antNo $=50$

rho $=0.8$

alpha $=1$

beta $=1$

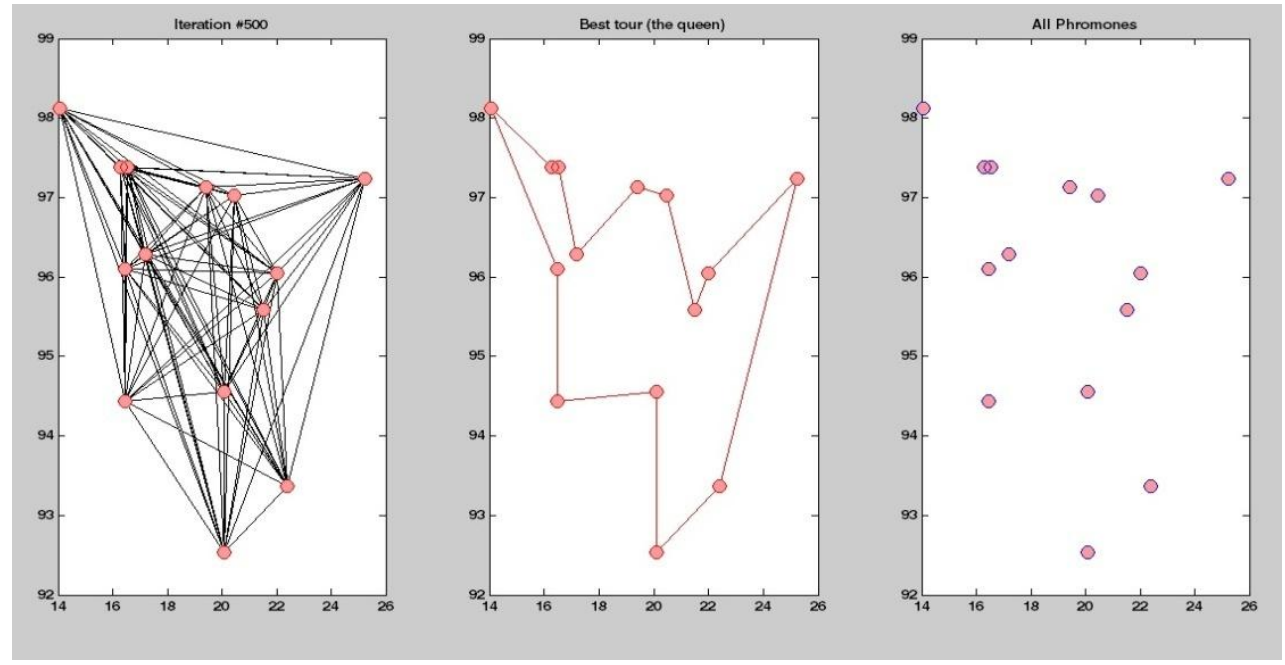

Fig7. The figure shows the formed iterations, best tour, and all pheromones as per reading of Case 4

The shortest length in case of 500 iterations with 50 ants at 0.8 evaporation rate comes out to be 30.8785 as shown in Fig 8.

Command Window
Iteration $\$ 487$ shortest length $=30.8785$
Iteration $\$ 488$ shortest length $=30.8785$
Iteration $\$ 489$ shortest length $=30.8785$
Iteration $\$ 490$ shortest length $=30.8785$
Iteration $\$ 491$ shortest length $=30.8785$
Iteration $\$ 492$ shortest length $=30.8785$
Iteration $\$ 493$ shortest length $=30.8785$
Iteration $\$ 494$ shortest length $=30.8785$
Iteration $\$ 495$ shortest length $=30.8785$
Iteration $\$ 496$ shortest length $=30.8785$
Iteration $\$ 497$ shortest length $=30.8785$
Iteration $\$ 498$ shortest length $=30.8785$
Iteration $\$ 499$ shortest length $=30.8785$
Iteration $\$ 500$ shortest length $=30.8785$

Fig8. The shortest length in case 4 of 500 iterations with 50 ants at evaporation 0.8 comes out to be 30.8785 


\section{Case 5}

maxIter $=100$

antNo $=50$

rho $=0.8$

alpha $=1$

beta $=1$

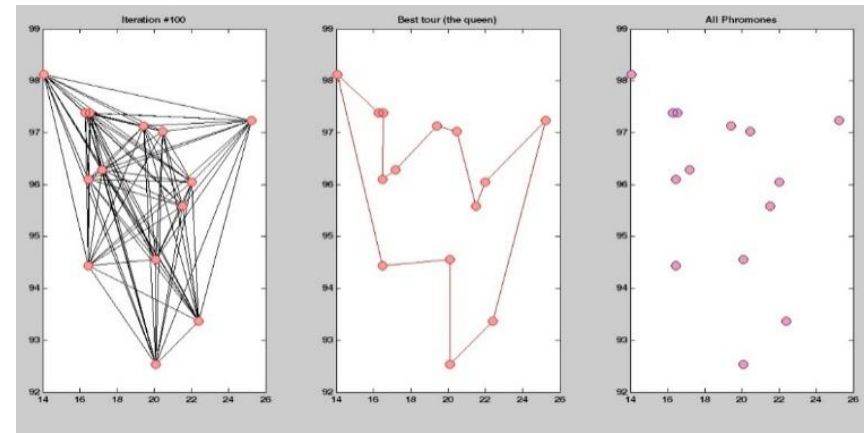

Fig9. The figure shows the formed iterations, best tour, and all pheromones as per reading of Case 5

The shortest length in case of 100 iterations with 50 ants at 0.8 evaporation rate comes out to be 31.2269 as sown in Fig 10.

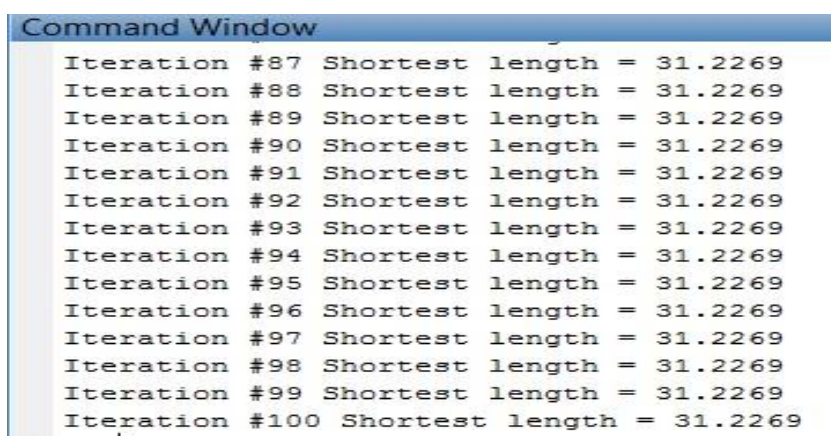

Fig10. The shortest length in case 5 of 100 iterations with 50 ants and 0.8 evaporation comes out to be 31.2269

\section{Case 6}

maxIter $=50$

$\operatorname{antNo}=50$

rho $=0.8$

alpha $=1$

beta $=1$

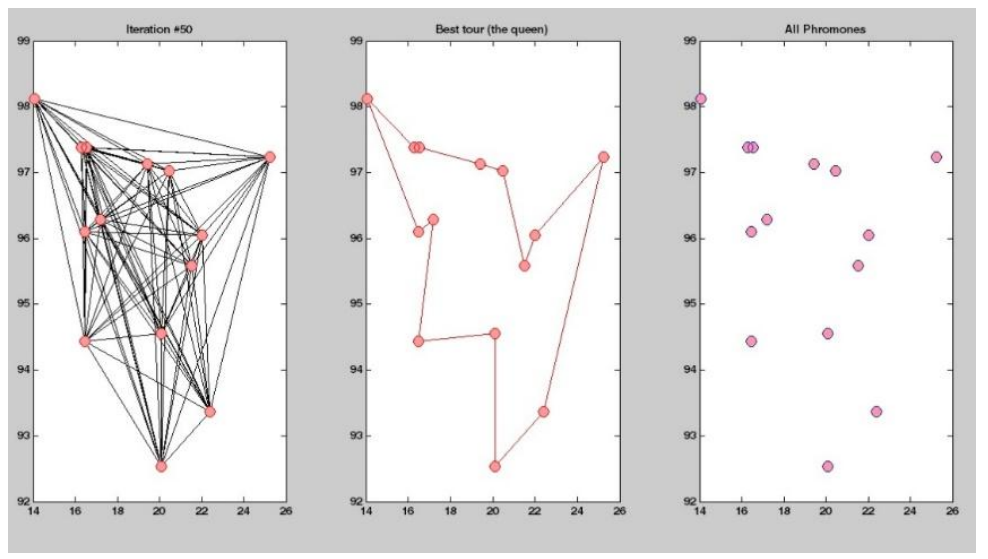

Fig11. The figure shows the formed iterations, best tour, and all pheromones as per reading of Case5 
The shortest length in case of 50 iterations with 50 ants at 0.8 evaporation rate comes out to be 31.2088 as shown in Fig. 12.

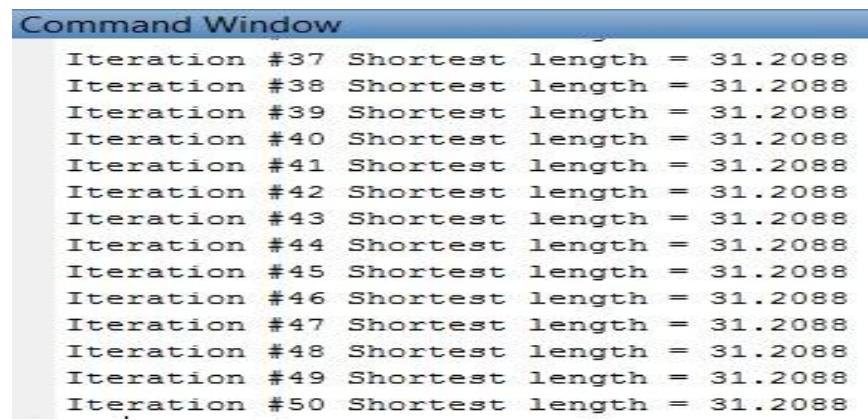

Fig12. The shortest length in case6 of 50 iterations with 50 ants and 0.8 evaporation comes out to be 31.2088

\section{Case7}

maxIter $=700$

antNo $=50$

rho $=0.5$

alpha $=1$

beta $=1$
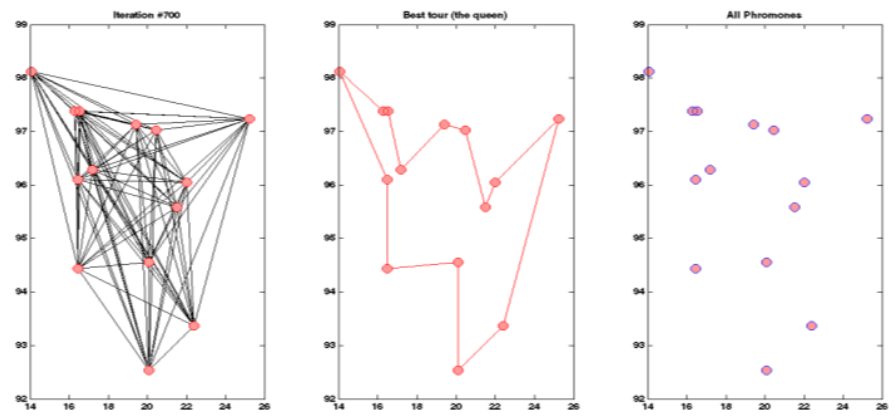

Fig13. The figure shows the formed iterations, best tour, and all pheromones as per reading of Case 7

The shortest length in case of 700 iterations with 50 ants at 0.5 evaporation rate comes out to be 30.8785 as shown in Fig. 14.

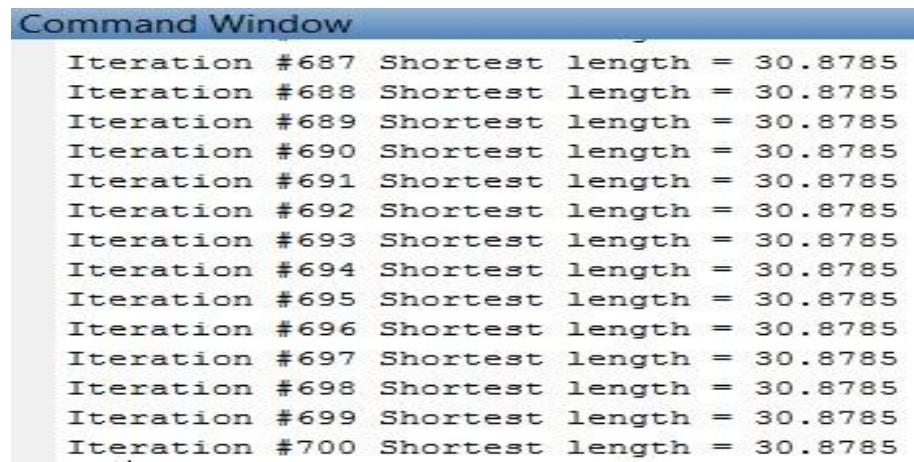

Fig14. The shortest length in case 7 of 700 iterations with 50 ants and 0.5 evaporation comes out to be 30.8785

\section{Case8}

maxIter $=700$

antNo $=50$

rho $=0.8$

alpha $=1$

beta $=1$ 

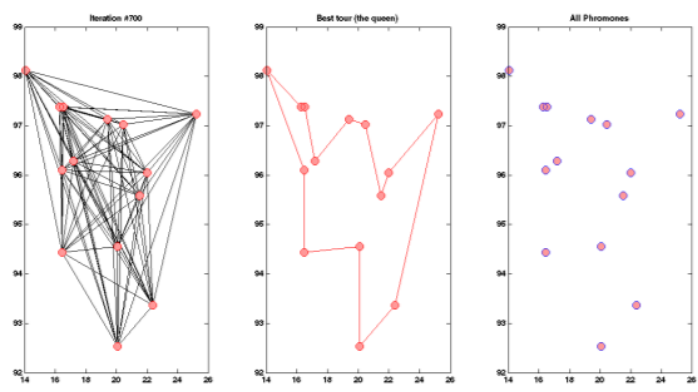

Fig15. The figure shows the formed iterations, best tour, and all pheromones as per reading of Cas 8

The shortest length in case of 700 iterations with 50 ants at 0.8 evaporation rate comes out to be 30.8785 as shown in Fig 16.

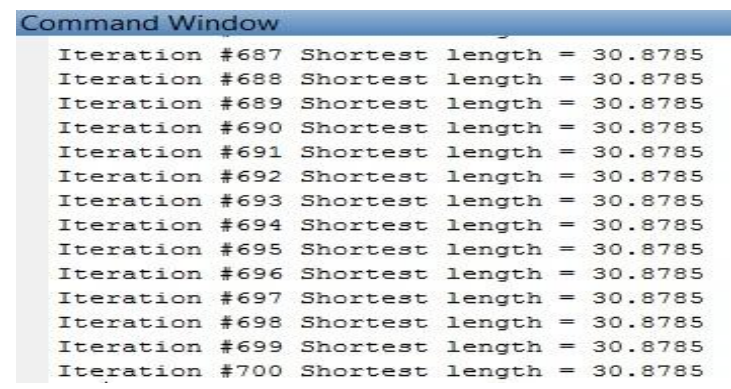

Fig16. The shortest length in case 8 of 700 iterations with 50 ants and 0.8 evaporation comes out to be 30.8785

\section{Case9}

maxIter $=1000$

$\operatorname{antNo}=50$

rho $=0.5$

alpha $=1$

beta $=1$
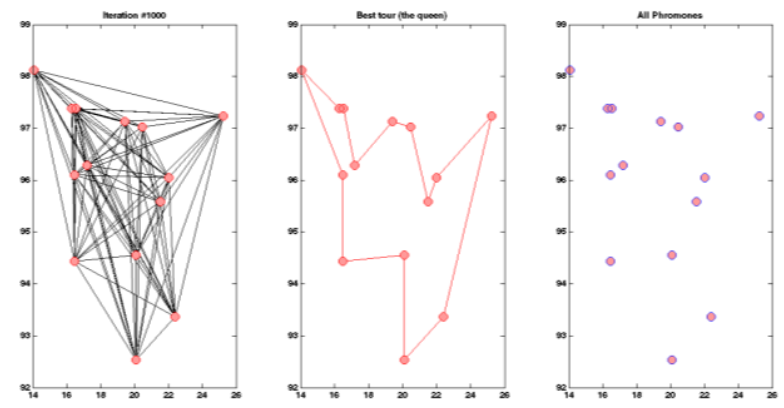

Fig17. The figure shows the formed iterations, best tour, and all pheromones as per reading of Case 9

The shortest length in case of 1000 iterations with 50 ants at 0.5 evaporation rate comes out to be 30.8785 as shown in Fig 18.

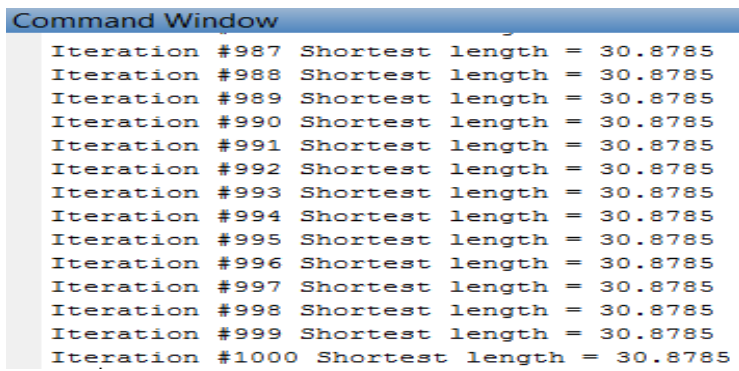

Fig18.The shortest length in case 9 of 1000 iterations with 50 ants and 0.5 evaporation comes out to be 30.8785 


\section{Case10}

maxIter $=1000$

antNo $=50$

rho $=0.8$

alpha $=1$

beta $=1$
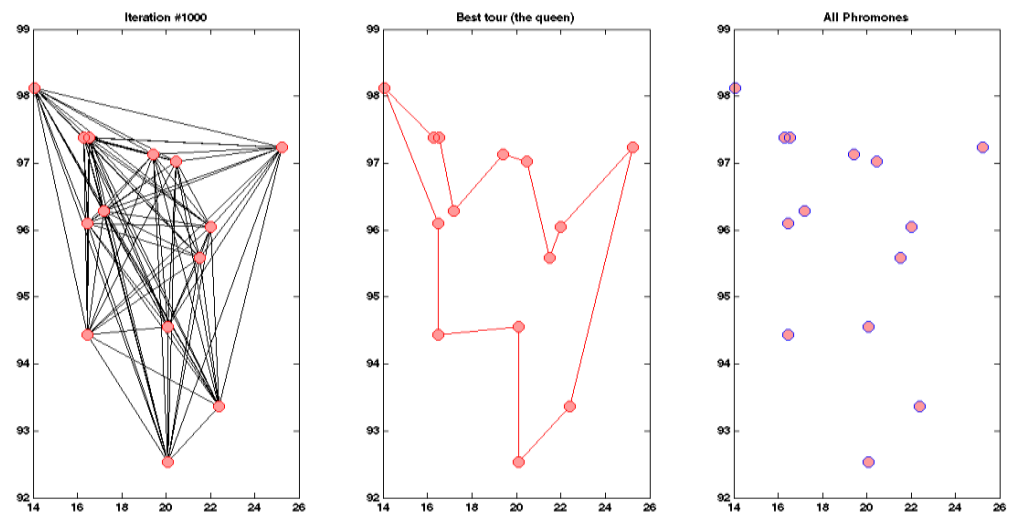

Fig19. The figure shows the formed iterations, best tour, and all pheromones as per reading of Case10

The shortest length in case of 1000 iterations with 50 ants at 0.8 evaporation rate comes out to be 30.8785 as shown in Fig 20.

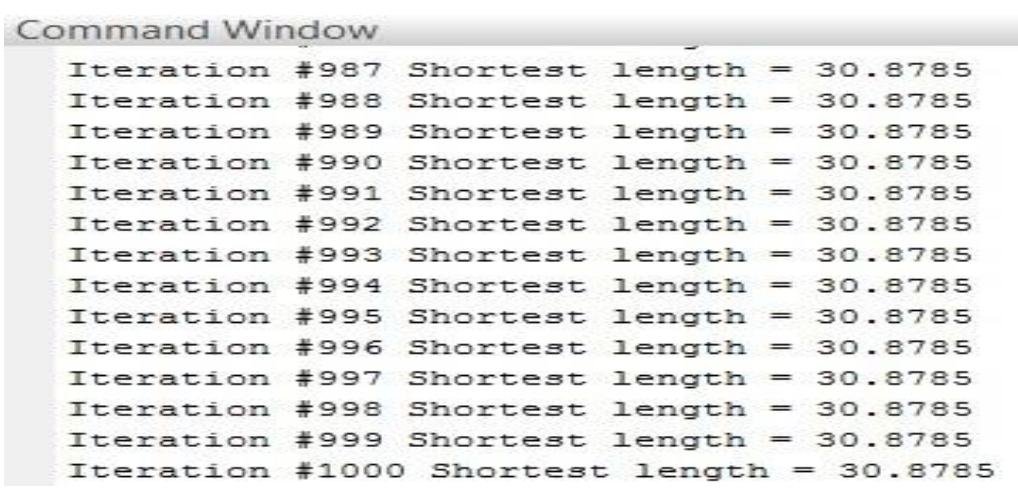

Fig20. The shortest length in case 10 of 1000 iterations with 50 ants and 0.8 evaporation comes out to be 30.8785

\section{Comparative Evaluation of Different CaSes under Study}

This section is intended to perform the comparatively analyzing the different cases studied in above section (Section II) of the research paper. The table has been constructed on the basis of findings calculated in section II of the research paper.

Table1.Comparative readings under different scenarios in $A C O$

\begin{tabular}{|l|l|l|l|l|}
\hline Case & maxIter & antNo & Rho & Shortest_Length \\
\hline $\mathbf{1}$ & $\mathbf{5 0 0}$ & $\mathbf{5 0}$ & $\mathbf{0 . 5}$ & $\mathbf{3 0 . 8 7 8 5}$ \\
\hline $\mathbf{2}$ & $\mathbf{1 0 0}$ & $\mathbf{5 0}$ & $\mathbf{0 . 5}$ & $\mathbf{3 0 . 8 7 8 5}$ \\
\hline $\mathbf{3}$ & $\mathbf{5 0}$ & $\mathbf{5 0}$ & $\mathbf{0 . 5}$ & $\mathbf{3 1 . 5 6 7}$ \\
\hline $\mathbf{4}$ & $\mathbf{5 0 0}$ & $\mathbf{5 0}$ & $\mathbf{0 . 8}$ & $\mathbf{3 0 . 8 7 8 5}$ \\
\hline $\mathbf{5}$ & $\mathbf{1 0 0}$ & $\mathbf{5 0}$ & $\mathbf{0 . 8}$ & $\mathbf{3 1 . 2 2 6 9}$ \\
\hline $\mathbf{6}$ & $\mathbf{5 0}$ & $\mathbf{5 0}$ & $\mathbf{0 . 5}$ & $\mathbf{3 1 . 2 0 8 8}$ \\
\hline $\mathbf{7}$ & $\mathbf{7 0 0}$ & $\mathbf{5 0}$ & $\mathbf{0 . 8}$ & $\mathbf{3 0 . 8 7 8 5}$ \\
\hline $\mathbf{8}$ & $\mathbf{7 0 0}$ & $\mathbf{5 0}$ & $\mathbf{0 . 5}$ & $\mathbf{3 0 . 8 7 8 5}$ \\
\hline $\mathbf{9}$ & $\mathbf{1 0 0 0}$ & $\mathbf{5 0}$ & $\mathbf{0 . 8}$ & $\mathbf{3 0 . 8 7 8 5}$ \\
\hline 10 & $\mathbf{1 0 0 0}$ & $\mathbf{5 0}$ & & $\mathbf{3 0 . 8 7 8 5}$ \\
\hline
\end{tabular}

Fig21shows the graphical representation of Table1. 


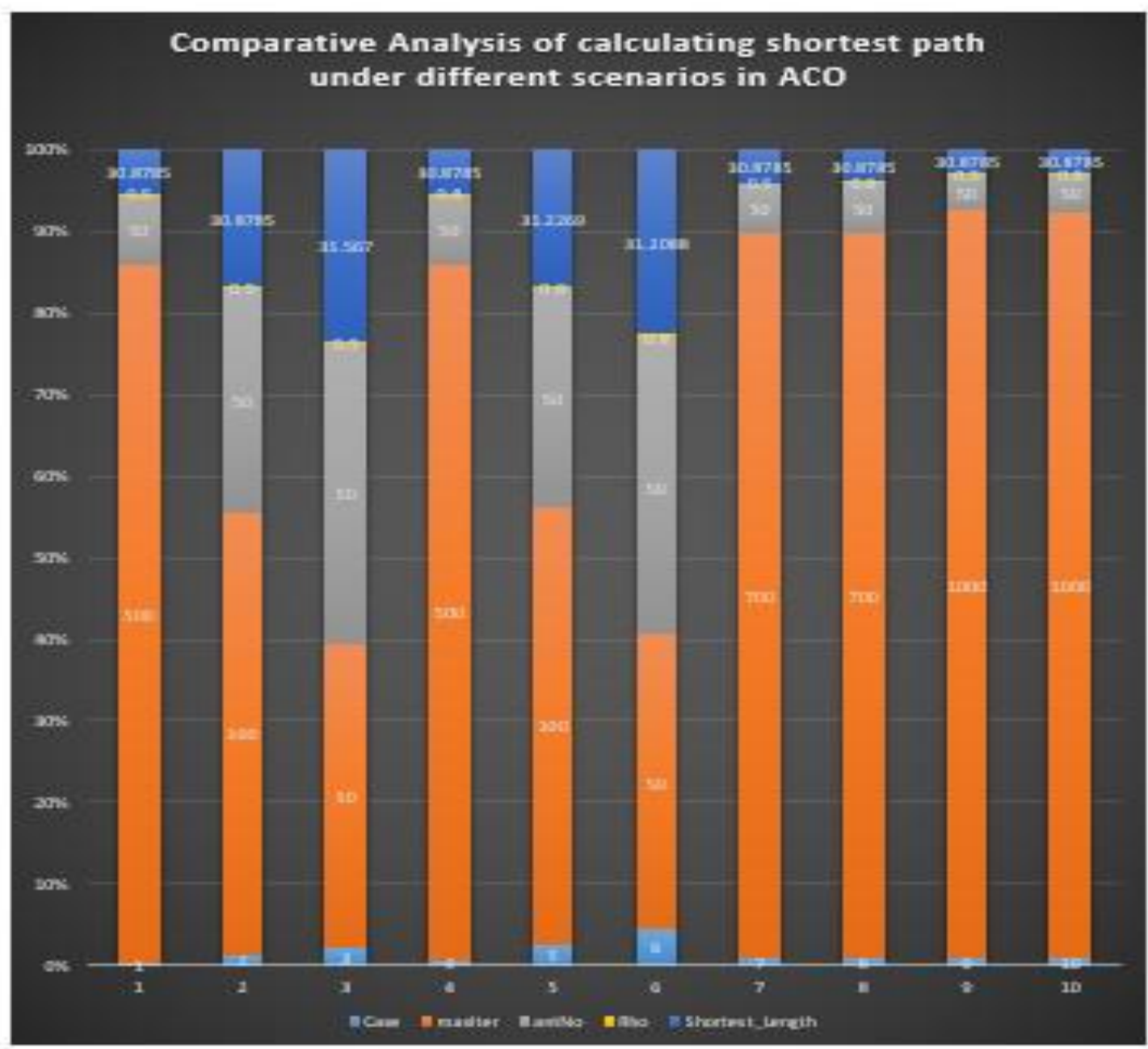

Fig21.The figure shows the graphical representation of Table1

\section{CONCLUSION}

On the basis of the conducted study and implementation, it can be concluded that the calculation of the shortest path depends upon several factors as used above in the implementation. On the basis of analyzing different results, it can be declared that as the number of the iterations increases, so the chances of finding the shortest path enhances.

\section{REFERENCES}

[1] Raghavendra BV, Solving Travelling Salesman Problem using Ant Colony Optimization Algorithm, J Appl Computat Math 2015, 4:6, http://dx.doi.org/10.4172/2168-9679.1000260

[2] M. Pedemonte, S. Nesmachnow, H. Cancela, A survey on parallel ant colony optimization, Applied Soft Computing, 11 (8) (2011), pp. 5181-5197.

[3] M. Pedemonte, H. Cancela, A cellular ant colony optimization for the generalized steiner problem, International Journal of Innovative Computing and Applications, 2 (3), (2010), pp. 188-201.

[4] M. Subotic, M. Tuba, N. Stanarevic, Different approaches in parallelization of the artificial bee colony algorithm, International Journal of Mathematical Models and Methods in Applied Sciences, 5 (4), (2011), pp. $755-762$.

[5] Jagdev, G. (2018). Scrutinizing and Execution of AODV and DVR Routing Protocols in Wireless Ad-hoc Networks. International Journal of Research Studies in Computer Science and Engineering (IJRSCSE), 5(3), pp.15-22. http://dx.doi.org/10.20431/ 2349-4859.0503003.

[6] Jagdev, G. (2018). Analyzing and Implementation of Ant Colony Optimization in Relevance with Travelling Salesman Problem. International Journal of Research Studies in Computer Science and Engineering (IJRSCSE), 5(4), pp.22-29. http://dx.doi.org/10.20431/2349-4859.0504003.

[7] J. Chintalapati, M. Arvind, S. Priyanka, N. Mangala, J. Valadi, Parallel ant-miner (pam) on high performance clusters, in: Swarm, Evolutionary, and Memetic Computing, Vol. 6466 of Lecture Notes on Computer Science, Springer, 2010, pp. 270-277. 
[8] S. Ilie, M. Ivanovic et al., Optimizing communication costs in acoda using simulated annealing: Initial experiments, in: N. T. Nguyen, K. Hoang, P. Jedrzejowicz (Eds.), Proc.4th International Conference o Computational Collective Intelligence. Technologies and applications, ICCCI 2012 (1), Vol. 7653 of Lecture Notes in Computer Science, Springer, 2012, pp. 298-307.

[9] Retno S and Rosita K 2015 Proc. Int. on Research and Education Mathematics vol.7 (Malaysia: Kuala Lumpur/ IEEE Xplore).

[10] Marco Dorigo and Luca Maria Gambardella. Ant colony system: a cooperative learning approach to the traveling salesman problem. IEEE Transactions on evolutionary computation, 1(1):53\{66, 1997.

[11] Alers S, Tuyls K, Ranjbar-Sahraei B, Claes D, and Weiss G. Insect-inspired robot coordination:Foraging and coverage. Arti_cial Life, 14:761-768, 2014.

[12] Alers S, Claes D, Tuyls K, and Weiss G. Biologically inspired multi-robot foraging. In Proceedings of the 2014 international conference on Autonomous agents and multi-agent systems, pages $1683\{1684$. International Foundation for Autonomous Agents and Multiagent Systems, 2014.

\section{AUTHOR'S BIOGRAPHY}

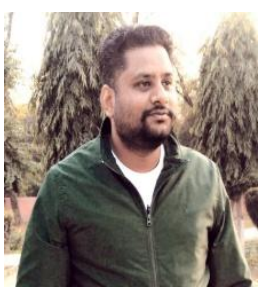

Dr. Gagandeep Jagdev, is working in the capacity of a faculty member in Dept. of Computer Science, Punjabi University Guru Kashi College, Damdama Sahib, Punjab, India. His total teaching experience is more than 12 years and has 132 international and national publications in reputed journals and conferences to his credit. He is also a member of editorial board of several international peerreviewed journals and has been active TPC member of several international and national conferences conducted by renowned universities and academic institutions. He chaired the sessions of several International Conferences conducted and sponsored by prominent academic bodies. He has been awarded Best Research Paper award at NCEEITET - 2015 held at Govt. College of Engineering \&Technology, Jammu. He has also been awarded with Best Research Paper Award by National Institute of Technical Teachers Training and Research (NITTTR), Chandigarh at International Conference ICCCN - 2017 conducted by Dept. of CSE. His field of expertise is Big Data, Data Mining, Image processing, ANN, Biometrics, RFID, Cloud Computing, Cloud security, Cryptography, MANETs, and VANETs.

Citation:Gagandeep, j. (2019). Evaluating Different Scenarios in Wanets to Find Shortest Path Applying ACO. International Journal of Research Studies in Computer Science and Engineering (IJRSCSE), 6(1), pp.48-57. http://dx.doi.org/10.20431/2349-4859.0601005

Copyright:@ 2018 Authors, This is an open-access article distributed under the terms of the Creative Commons Attribution License, which permits unrestricted use, distribution, and reproduction in any medium, provided the original author and source are credited. 